\section{Commentary: Proving the benefit of pulmonary metastasectomy for relapsed osteosarcoma - to the next hard target?}

\author{
Benjamin Wei, MD, and Tyler S. Wahl, MD, MSPH
}

Osteosarcoma is a devastating primary malignant bone tumor mostly affecting younger patients, with many presenting with pulmonary metastases at the time of diagnosis. ${ }^{1}$ Liu and colleagues, ${ }^{2}$ in their study, "Survival After Pulmonary Metastasectomy for Relapsed Osteosarcoma," present data that support pulmonary metastasectomy among patients with limited relapse following initial therapy with R0 resection and adjuvant chemotherapy. The investigators reported a greater postrelapse survival in the metastasectomy group compared with the nonsurgical group $(68.4 \%$ vs $25 \%$ at 2 years; $41 \%$ vs $0 \%$ at 5 years). Note that patients who underwent metastasectomy in the current study presented with longer relapse-free intervals, lower relapse rates during initial therapy, and lower alkaline phosphatase serum levels at time of relapse than the nonsurgical group. This highlights an important concept of the "oncologic stress test": selecting metastasectomy in patients with more favorable tumor biology.

Of course, one common argument "against" metastasectomy has exactly to do with this point: that selection bias distorts survival data. Parrying this argument, the investigators have performed a propensity-matched analysis to determine whether a survival benefit to this specific operation in this specific group of patients truly exists. Indeed, they

\footnotetext{
From the Division of Cardiothoracic Surgery, University of Alabama at Birmingham Medical Center, Birmingham, Ala.

Disclosures: The authors reported no conflicts of interest.

The Journal policy requires editors and reviewers to disclose conflicts of interest and to decline handling or reviewing manuscripts for which they may have a conflict of interest. The editors and reviewers of this article have no conflicts of interest.

Received for publication Nov 10, 2020; revisions received Nov 10, 2020; accepted for publication Nov 13, 2020; available ahead of print Nov 19, 2020.

Address for reprints: Benjamin Wei, MD, Division of Cardiothoracic Surgery, University of Alabama at Birmingham, Zeigler Research Building 701, 1720 2nd Ave South, Birmingham, AL 35294 (E-mail: bwei@uab.edu).

J Thorac Cardiovasc Surg 2022;163:480-1

0022-5223/\$0.00

Published by Elsevier Inc. on behalf of The American Association for Thoracic Surgery

https://doi.org/10.1016/j.jtcvs.2020.11.043
}

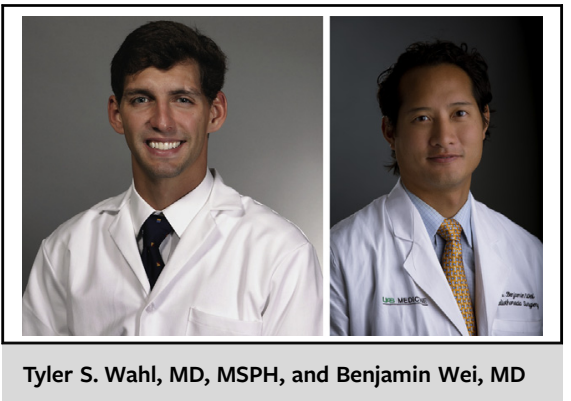

\author{
CENTRAL MESSAGE \\ Pulmonary metastasectomy ap- \\ pears to impart improved post- \\ relapse survival compared with \\ chemotherapy alone in patients \\ with relapsed osteosarcoma \\ following initial Ro resection.
}

demonstrate a decreased hazard ratio ranging from 0.14 to 0.22 of metastasectomy for postrelapse survival $(P<.001)$. Now, do eleven different methods of propensity matching add up to a single randomized controlled trial? Likely not, but given that the latter have been notoriously difficult to accrue, this article answers the question as convincingly as any. ${ }^{3}$

In this study, $22 \%$ of patients relapsed a second time despite undergoing complete R0 metastasectomy, but only 4 of 20 of these patients then underwent a second metastasectomy; yet, there is growing support for repeated metastasectomy among patients with sarcoma, osteosarcoma included. ${ }^{4,5}$ In addition, there were differences related to the timing of chemotherapy among patients who underwent metastasectomy. The question remains: what is the optimal sequence of second-line adjuvant chemotherapy and metastasectomy for patients who relapse? Larger, multi-institutional prospective studies may address this key question, as the existing literature is limited by heterogeneity in patient inclusion criteria and widely variable sample sizes and practice locales. Such larger studies may yield a risk calculator or build on current nomograms fashioned from smaller cohorts. ${ }^{6,7}$ Finally, given the emerging role of stereotactic radiosurgery in the treatment of oligometastatic disease, a comparison between surgical metastasectomy and "radiometastasectomy" may 
become the more salient question in the future, rather than additional comparisons between surgical and medical therapies. ${ }^{8}$

\section{References}

1. Aljubran AH, Griffin A, Pintilie M, Blackstein M. Osteosarcoma in adolescents and adults: survival analysis with and without lung metastases. Ann Oncol. 2009;20:1136-41.

2. Liu Z, Yin J, Zhou Q, Yang J, Zeng B, Yeung S-CJ, et al. Survival after pulmonary metastasectomy for relapsed osteosarcoma. J Thorac Cardiovasc Surg. 2022;163: 469-79.e8.

3. Treasure T, Farewell V, Macbeth F, Monson K, Williams NR, Brew-Graves C, et al. Pulmonary metastasectomy versus continued active monitoring in colorectal cancer (PulMICC): a multicentre randomized clinical trial. Trials. 2019;20:718.
4. Ramanujan V, Krishnamurthy A, Venkataramani K, Kumar C. Pulmonary metastasectomy in primary extremity osteosarcoma: choosing wisely, along with a brief review of literature. Indian J Cancer. 2020;57:172-81.

5. Li Y, Zhang W, Li S, Tu C. Clinical efficiency of repeated pulmonary metastasectomy in sarcoma patients with recurrent pulmonary metastasis: a meta-analysis. J Cancer Res Ther. 2018;14(suppl):S457-62.

6. Lin AY, Kotova S, Yanagawa J, Elbuluk O, Wang G, Kar N, et al. Risk stratification of patients undergoing pulmonary metastasectomy for soft tissue and bone sarcomas. J Thorac Cardiovasc Surg. 2015;149:85-92.

7. Wang B, Tu J, Yin J, Zou C, Wang J, Huang G, et al. Development and validation of a pretreatment prognostic index to predict death and lung metastases in extremity osteosarcoma. Oncotarget. 2015;6:38348-59.

8. Dhakal S, Corbin KS, Milano MT, Philip A, Sahasrabudhe D, Jones C, et al. Stereotactic body radiotherapy for pulmonary metastases from soft-tissue sarcomas: excellent local lesion control and improved patient survival. Int J Radiol Oncol Biol Phys. 2012;82:940-5.

\section{Commentary: Upping our game}

\section{Todd L. Demmy, MD}

In this issue of the Journal, Liu and colleagues ${ }^{1}$ report a useful large case series of osteosarcoma patients who underwent resection of their lung metastases. Fewer involved lobar regions (rather than just absolute numbers) and lower alkaline phosphatase levels were nuanced advantages that should be studied by others for replication. Like other thoughtful investigators, the authors recommend a prospective trial to validate their findings.

Is a randomized study the correct next step for a complex diverse disease for which we have new tools for study? Patients receive benefit, suffer harm, or break even from our attempts at local resection, depending on their sarcoma biologies. Aggressive tumors beat surgical therapies, and some experts argue that patients with indolent sarcoma might live just as long without operative interference. ${ }^{2}$

Most surgeons have seen metastatic disease accelerate after cancer operations because of inflammatory cytokines, stimulation of angiogenesis, or removal of tumor-related

\footnotetext{
From the Department of Thoracic Surgery, Roswell Park Cancer Institute, Buffalo, New York and Department of Surgery, University at Buffalo, Buffalo, NY. Disclosures: Dr Demmy has served as a consultant for Medtronic.

The Journal policy requires editors and reviewers to disclose conflicts of interest and to decline handling or reviewing manuscripts for which they may have a conflict of interest. The editors and reviewers of this article have no conflicts of interest.

Received for publication Nov 17, 2020; revisions received Nov 17, 2020; accepted for publication Nov 18, 2020; available ahead of print Nov 28, 2020.

Address for reprints: Todd L. Demmy, MD, Department of Thoracic Surgery, Roswell Park Cancer Institute, Elm and Carlton Sts, Buffalo, NY 14263 (E-mail: todd. demmy@ roswellpark.org).

J Thorac Cardiovasc Surg 2022;163:481-2

$0022-5223 / \$ 36.00$

Copyright (c) 2020 by The American Association for Thoracic Surgery

https://doi.org/10.1016/j.jtcvs.2020.11.086
}

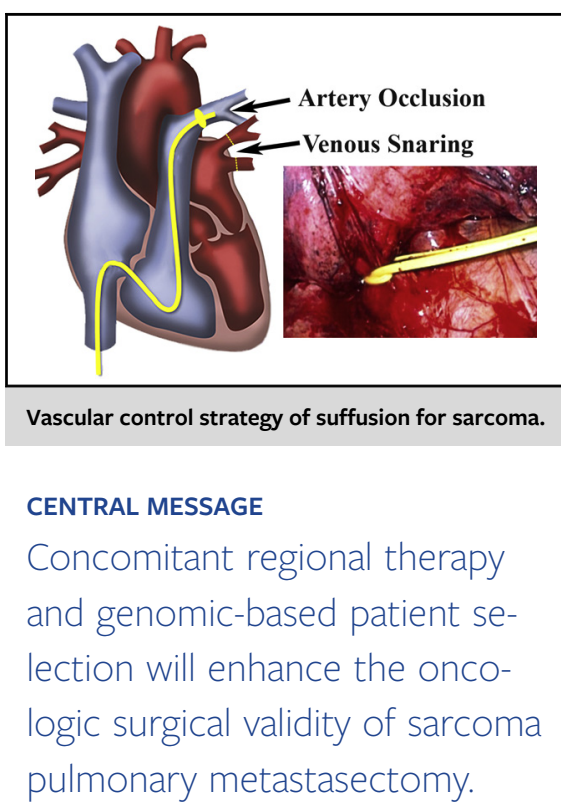

inhibitory factors. ${ }^{3,4}$ However, even when appropriately counseled regarding potentially unfavorable recurrence dynamics, patients face a psychologic imperative to accept surgery rather than watching their disease progress as other options fail. This imperative would likely be a larger barrier to randomized investigations than observed when comparing open operations with minimally invasive operations. Perhaps patients would agree to be randomized between immediate or delayed surgical approaches to test a noninferiority hypothesis. However, would such hypotheses, if proven, provide sufficient evidence to change practice for patients with few other optimistic options? 